\title{
On boundary-value problems for partial differential equations of order higher than two
}

\author{
by JAN PopIOŁEK (Białystok)
}

\begin{abstract}
We prove the existence of solutions of some boundary-value problems for partial differential equations of order higher than two. The general idea is similar to that in [1]. We make an essential use of the results of our paper [12].
\end{abstract}

1. The problem. Let $x=\chi_{p}(t), 0<t \leq T, p=1,2$, be equations of non-intersecting curves on the $(x, t)$ plane.

In this paper we prove the existence of a solution of the problem

$$
\mathcal{L} u(x, t) \equiv \sum_{i=0}^{n+2} \sum_{j=0}^{m} a_{i j}(x, t) D_{x}^{i} D_{t}^{j} u(x, t)-D_{x}^{n} D_{t}^{m+1} u(x, t)=f(x, t),
$$

where $(x, t) \in \mathbf{S}_{T}=\left\{(x, t): \chi_{1}(t)<x<\chi_{2}(t), 0<t \leq T\right\}, T=$ const $<\infty$, $n, m \in \mathbb{N}_{0} \equiv \mathbb{N} \cup\{0\}, n+m>0$ (for $n=m=0$ equation (1) is a parabolic equation of second order, the theory of which is well known), satisfying the initial conditions

$$
D_{t}^{l} u(x, 0)=0, \quad \chi_{1}(0) \leq x \leq \chi_{2}(0), l=0,1, \ldots, m,
$$

and the boundary conditions

$$
\mathbf{B}_{l}^{p} u\left(\chi_{p}(t), t\right) \equiv \sum_{k=0}^{r_{l}^{p}} b_{k l}^{p}(t) D_{x}^{k} u\left(\chi_{p}(t), t\right)=\mathbf{g}_{l}^{p}(t),
$$

where $0<t \leq T, p=1,2, l=1, \ldots, l_{0}=[(n+3) / 2]$ (denotes the greatest integer function), $0 \leq r_{1}^{p}<r_{2}^{p}<\ldots<r_{l_{0}}^{p} \leq n+1, r_{l}^{p} \in \mathbb{N}_{0}, b_{r_{l}^{p}, l}^{p}(t) \geq b_{0}=$ const $>0$.

We distinguish the following four cases:

1) $r_{l_{0}}^{p}<n+1, p=1$ or $p=2, n$ is odd,

1991 Mathematics Subject Classification: Primary 35G15; Secondary 45D05.

Key words and phrases: partial differential equation, boundary-value problem, Volterra integral equation. 
2) $r_{l_{0}}^{p}<n+1, p=1$ or $p=2, n$ is even,

3) $r_{l_{0}}^{p}=n+1, p=1$ or $p=2, n$ is odd,

4) $r_{l_{0}}^{p}=n+1, p=1$ or $p=2, n$ is even.

We shall exactly analyse cases 1 ) and 3 ). The argument in the remaining cases is similar. Note that in cases 1$)$ and 3) we have to put $[(n-1) / 2]$ boundary conditions on one of the curves $\chi_{p}$ and $[(n-1) / 2]+1$ on the other.

Boundary-value problems in rectangular domains and for particular cases of the operator $\mathcal{L}$ and of the boundary operators $\mathbf{B}_{l}^{p}$ have been considered in many papers (see [2], [3], [4], [10] and [15]). In [14] the boundary-value problem for the equation

$$
D_{x}^{n+2} u-D_{x}^{n} D_{t} u=f\left(x, t, u, \ldots, D_{x}^{n+1} u\right)
$$

was examined. Paper [13] was devoted to the equation

$$
\mathbf{L}\left(D_{x}+D_{t}\right)^{n} u(x, t)=f(x, t),
$$

where $\mathbf{L} \equiv D_{t}-a(x, t) D_{x}^{2}+b(x, t) D_{x}+c(x, t)$. In [5] some boundary-value problems for the equation

$$
\left(D_{x}^{2}-D_{t}\right)\left(a D_{x}+b D_{t}+c\right) u(x, t)=0
$$

were investigated, where $a, b, c$ are constants and $a \cdot b \neq 0$. Moreover, in [11] Cauchy's problem for equation (1) was examined.

Note that particular cases of equation (1) describe the propagation of waves in a compressible viscous medium (see [3], [6], [17]) and some problems of magneto-hydrodynamics (see [8], [9]).

2. Assumptions. We make the following assumptions:

(A.1) There are constants $a_{0}$ and $a_{1}$ such that

$$
0<a_{0} \leq a_{n+2, m}(x, t) \leq a_{1} \quad \text { for }(x, t) \in \overline{\mathbf{S}}_{T}
$$

$\left(\overline{\mathbf{S}}_{T}\right.$ denotes the closure of $\left.\boldsymbol{S}_{T}\right)$.

(A.2) The coefficients $a_{i j}(i=0,1, \ldots, n+2, j=0,1, \ldots, m)$ are continuous in $\overline{\mathbf{S}}_{T}$ and satisfy the Hölder condition with respect to $x$ with exponent $\alpha(0<\alpha \leq 1)$; moreover, $a_{n+2, m}$ satisfies the Hölder condition with respect to $t$ with exponent $\frac{1}{2} \alpha$.

(A.3) The functions $\chi_{p}(p=1,2)$ have continuous derivatives up to order $n_{*}=[(n+1) / 2]$ in $[0, T]$ and the highest derivatives satisfy the Hölder condition

$$
\left|\Delta_{t}\left[\chi_{p}^{\left(n_{*}\right)}(t)\right]\right| \leq \mathrm{const} \begin{cases}(\Delta t)^{\alpha / 2} & \text { if } n+1 \text { is even, } \\ (\Delta t)^{(\alpha+1) / 2} & \text { if } n+1 \text { is odd, }\end{cases}
$$

where $\Delta_{t}[\chi(t)] \equiv \chi(t+\Delta t)-\chi(t), t, t+\Delta t \in[0, T], \alpha \in(0,1]$. 
(A.4) The function $f(x, t)$ is defined and continuous for $(x, t) \in \mathbf{S}_{T}$, and satisfies the inequalities

$$
|f(x, t)| \leq M_{f}, \quad\left|\Delta_{x} f(x, t)\right| \leq m_{f}|\Delta x|^{\alpha},
$$

where $\Delta_{x} f(x, t) \equiv f(x+\Delta x, t)-f(x, t),(x, t),(x+\Delta x, t) \in \overline{\mathbf{S}}_{T}, M_{f}$, $m_{f}=$ const $>0, \alpha \in(0,1]$.

(A.5) The functions $\mathbf{g}_{l}^{p}, p=1,2, l=1, \ldots, l_{0}$, are defined and have continuous derivatives $D_{t}^{\nu} \mathbf{g}_{l}^{p}\left(\nu=0,1, \ldots, \mathcal{M}=\left[d_{r} / 2\right], d_{r}=n-r_{l}^{p}+2 m+1\right)$ in $[0, T]$ and satisfy the conditions

$$
\left|\Delta_{t}\left[D_{t}^{\mathcal{M}} \mathbf{g}_{l}^{p}(t)\right]\right| \leq M_{g} \begin{cases}(\Delta t)^{\alpha / 2} & \text { if } d_{r} \text { is even, } \\ (\Delta t)^{(\alpha+1) / 2} & \text { if } d_{r} \text { is odd }\end{cases}
$$

and $D_{t}^{\nu} \mathbf{g}_{l}^{p}(0)=0$, where $M_{g}=$ const $>0,0<\alpha \leq 1$.

(A.6) The functions $b_{k l}^{p}, p=1,2, l=1, \ldots, l_{0}, k=0,1, \ldots, r_{l}^{p}$, are defined in $[0, T]$ and have continuous derivatives up to order $\mathcal{M}$.

R e m a r k. Without restricting generality, we can assume $b_{r_{l}^{p}, l}^{p}(t) \geq b_{0} \equiv 1$.

3. Solution of the problem. In all cases 1)-4) we shall seek a solution of the problem (1)-(3) in the form

$$
u(x, t)=\sum_{\sigma=1}^{2} \sum_{q=1}^{l_{0}} \int_{0}^{t} \Lambda_{r_{q}^{\sigma}}\left(x, t ; \chi_{\sigma}(\tau), \tau\right) \varphi_{q}^{\sigma}(\tau) d \tau+\mathbf{Z}_{\mathbf{S}_{T}}(x, t),
$$

where $\varphi_{q}^{\sigma}$ are unknown functions, $\Lambda_{r_{q}^{\sigma}}$ are the fundamental solutions of (1) constructed in [12] and

$$
\mathbf{Z}_{\mathbf{S}_{T}}(x, t)=\iint_{\mathbf{S}_{t}} \Lambda_{0}(x, t ; y, \tau) f(y, \tau) d y d \tau
$$

3.1. Cas e 1). Observe that the function $u$ given by (4) satisfies equation (1) and initial conditions (2). Boundary conditions (3) lead to the system of equations

$$
\mathbf{g}_{l}^{p}(t)=\sum_{\sigma=1}^{2} \sum_{q=1}^{l_{0}} \int_{0}^{t} \mathbf{B}_{l}^{p} \Lambda_{r_{q}^{\sigma}}\left(\chi_{p}(t), t ; \chi_{\sigma}(\tau), \tau\right) \varphi_{q}^{\sigma}(\tau) d \tau+z_{l}^{p}(t),
$$

where $\boldsymbol{z}_{l}^{p}(t)=\mathbf{B}_{l}^{p} \mathbf{Z}_{\mathbf{S}_{T}}\left(\chi_{p}(t), t\right), 0<t \leq T, p=1,2, l=1, \ldots, l_{0}$.

By Lemma 3 of [12] we obtain

$$
\begin{aligned}
& D_{x}^{r_{l}^{p}} w_{r_{q}^{p}}\left(\chi_{p}(\tau), t ; \chi_{p}(\tau), \tau\right) \\
& \quad= \begin{cases}0, & 1 \leq l<q, \\
(-1)^{n-r_{l}^{p}} \sqrt{\pi}[\mathbf{a}(\tau)]^{\left(n-r_{l}^{p}\right) / 2} \Gamma^{-1}\left(d_{r} / 2\right)(t-\tau)^{d_{r} / 2-1}, & q \leq l \leq l_{0},\end{cases}
\end{aligned}
$$

$\left(p=1,2, l, q=1, \ldots, l_{0}\right)$, where $d_{r}=n-r_{l}^{p}+2 m+1$ and the functions $w_{r_{l}^{p}}$ are defined by formula (6) of [12], and $\mathbf{a}(\tau)=a_{n+2, m}\left(\chi_{p}(\tau), \tau\right)$. 
Using the definition of the operator $\mathbf{I}_{\kappa}([12],(25))$ and (7) we can write

(8) $\quad \int_{0}^{t} D_{x}^{r_{l}^{p}} w_{r_{q}^{p}}\left(\chi_{p}(\tau), t ; \chi_{p}(\tau), \tau\right) \varphi_{q}^{p}(\tau) d \tau=c_{l q}^{p} \mathbf{I}_{d_{r} / 2}\left([\mathbf{a}(t)]^{\left(n-r_{l}^{p}\right) / 2} \varphi_{q}^{p}(t)\right)$ $\left(p=1,2, l, q=1, \ldots, l_{0}, 0<t \leq T\right)$, where

$$
c_{l q}^{p}= \begin{cases}0, & 1 \leq l<q, \\ (-1)^{n-r_{l}^{p}} \sqrt{\pi}, & q \leq l \leq l_{0} .\end{cases}
$$

By (8) and (9) we can rewrite system (6) in the form

$$
\begin{aligned}
\sum_{q=1}^{l_{0}} c_{l q}^{p} \mathbf{I}_{d_{r} / 2}\left([\mathbf{a}(t)]^{\left(n-r_{l}^{p}\right) / 2} \varphi_{q}^{p}(t)\right) & \\
& +\sum_{\sigma=1}^{2} \sum_{q=1}^{l_{0}} \int_{0}^{t} \mathbf{K}_{l q}^{p \sigma}(t, \tau) \varphi_{p}^{\sigma}(\tau) d \tau+\boldsymbol{z}_{l}^{p}(t)=\mathbf{g}_{l}^{p}(t),
\end{aligned}
$$

where

$$
\begin{aligned}
& \mathbf{K}_{l q}^{p \sigma}(t, \tau)=\mathbf{B}_{l}^{p} \Lambda_{r_{q}^{\sigma}}\left(\chi_{p}(t), t \chi_{p}(\tau), \tau\right) \\
& \quad- \begin{cases}0 & \text { if } \sigma \neq p \text { or } \sigma=p \text { and } 1 \leq l<q, \\
D_{x}^{r_{l}^{p}} w_{r_{q}^{p}}\left(\chi_{p}(\tau), t ; \chi_{p}(\tau), \tau\right) & \text { if } \sigma=p \text { and } q \leq l \leq l_{0},\end{cases}
\end{aligned}
$$

$\left(p, \sigma=1,2, l, q=1, \ldots, l_{0}, 0<t \leq T\right)$.

(10) is a system of first-kind Volterra equations. Using the method given by Baderko [1] and the properties of the operator $\mathbf{R}_{1 / 2}$ defined by formula (14) of [12], we reduce this system to a system of second-kind Volterra equations. Applying to both sides of (10) the operator $\mathbf{R}_{1 / 2}^{d_{r}}$, where $d_{r}=$ $n-r_{l}^{p}+2 m+1$, by Lemma 4 of [12], we obtain

$$
\begin{aligned}
& \sum_{q=1}^{l_{0}} c_{l q}^{p}[\mathbf{a}(t)]^{\left(n-r_{l}^{p}\right) / 2} \varphi_{q}^{p}(t)+\sum_{\sigma=1}^{2} \sum_{q=1}^{l_{0}} \mathbf{R}_{1 / 2}^{d_{r}}\left[\int_{0}^{t} \mathbf{K}_{l q}^{p \sigma}(t, \tau) \varphi_{q}^{\sigma}(\tau) d \tau\right] \\
& +\mathbf{R}_{1 / 2}^{d_{r}}\left[\boldsymbol{z}_{l}^{p}(t)\right]=\mathbf{R}_{1 / 2}^{d_{r}}\left[\mathbf{g}_{l}^{p}(t)\right], \quad p=1,2, l=1, \ldots, l_{0}, 0<t \leq T .
\end{aligned}
$$

By Theorem 1 of [12],

$$
\left|D_{t}^{\nu} \mathbf{K}_{l q}^{p \sigma}(t, \tau)\right| \leq \mathrm{const}(t-\tau)^{\left(d_{r}-2 \nu+\alpha\right) / 2-1}
$$

$\left(\nu=0,1, \ldots, \mathcal{M}=\left[d_{r} / 2\right], d_{r}=n-r_{l}^{p}+2 m+1, p, \sigma=1,2, l, q=1, \ldots, l_{0}\right.$, $0 \leq \tau<t \leq T, 0<\alpha \leq 1)$.

We consider two cases: (i) $d_{r}$ is even, (ii) $d_{r}$ is odd.

In case (i) the function $\mathbf{K}_{l q}^{p \sigma}$ satisfies condition (18) of Lemma 4 of [12] with $N=d_{r} / 2$ and $\varrho=\alpha / 2$; hence, in view of formula (19) of [12] we have

$$
\mathbf{R}_{1 / 2}^{d_{r}}\left[\int_{0}^{t} \mathbf{K}_{l q}^{p \sigma}(t, \tau) \varphi_{q}^{\sigma}(\tau) d \tau\right]=\int_{0}^{t} D_{t}^{d_{r} / 2} \mathbf{K}_{l q}^{p \sigma}(t, \tau) \varphi_{q}^{\sigma}(\tau) d \tau .
$$


In case (ii), $\mathbf{K}_{l q}^{p \sigma}$ satisfies the same condition with $N=\left(d_{r}-1\right) / 2$ and $\varrho=(\alpha+1) / 2$; hence, by formula (20) of [12] we get

$$
\mathbf{R}_{1 / 2}^{d_{r}}\left[\int_{0}^{t} \mathbf{K}_{l q}^{p \sigma}(t, \tau) \varphi_{q}^{\sigma}(\tau) d \tau\right]=\int_{0}^{t} \boldsymbol{R}_{1 / 2}\left[D_{t}^{d_{r} / 2} \mathbf{K}_{l q}^{p \sigma}(t, \tau)\right] \varphi_{q}^{\sigma}(\tau) d \tau .
$$

By (14) and (15) system (12) can be written in the form

$$
\begin{aligned}
\sum_{q=1}^{l_{0}} c_{l q}^{p}[\mathbf{a}(t)]^{\left(n-r_{l}^{p}\right) / 2} & \varphi_{q}^{p}(t) \\
& +\sum_{\sigma=1}^{2} \sum_{q=1}^{l_{0}} \int_{0}^{t} \overline{\mathbf{K}}_{l q}^{p \sigma}(t, \tau) \varphi_{q}^{\sigma}(\tau) d \tau+\overline{\mathbf{z}}_{l}^{p}(t)=\overline{\mathbf{g}}_{l}^{p}(t)
\end{aligned}
$$

$\left(p=1,2, l=1, \ldots, l_{0}, 0<t \leq T\right)$, where

$$
\begin{gathered}
\overline{\mathbf{K}}_{l q}^{p \sigma}(t, \tau)= \begin{cases}D_{t}^{d_{r} / 2} \mathbf{K}_{l q}^{p \sigma}(t, \tau) & \text { if } d_{r} \text { is even, } \\
\mathcal{R}_{1 / 2}\left[D_{t}^{\left(d_{r}-1\right) / 2} \mathbf{K}_{l q}^{p \sigma}(t, \tau)\right] & \text { if } d_{r} \text { is odd, }\end{cases} \\
\overline{\boldsymbol{z}}_{l}^{p}(t)=\mathbf{R}_{1 / 2}^{d_{r}}\left[\boldsymbol{z}_{l}^{p}(t)\right], \\
\overline{\mathbf{g}}_{l}^{p}(t)=\mathbf{R}_{1 / 2}^{d_{r}}\left[\mathbf{g}_{l}^{p}(t)\right] .
\end{gathered}
$$

Now, we estimate the functions $\overline{\mathbf{K}}_{l q}^{p \sigma}, \overline{\mathbf{z}}_{l}^{p}$ and $\overline{\mathbf{g}}_{l}^{p}$. In case (i), by Theorem 1 [12], we have

$$
\begin{gathered}
\left|D_{t}^{d_{r} / 2} \mathbf{K}_{l q}^{p \sigma}(t, \tau)\right| \leq \mathrm{const}(t-\tau)^{\alpha / 2-1}, \quad 0 \leq \tau<t \leq T, \\
\left|\Delta_{t} D_{t}^{d_{r} / 2} \mathbf{K}_{l q}^{p \sigma}(t, \tau)\right| \leq \mathrm{const}(\Delta t)^{\beta / 2}(t-\tau)^{\mu-1},
\end{gathered}
$$

$0 \leq \tau<t \leq t+\Delta t \leq T, 0<\beta \leq \alpha \leq 1, \mu=\min \{\alpha / 2,1-\alpha / 2\}$.

Analogously, in case (ii), we get

$$
\begin{gathered}
\left|D_{t}^{\left(d_{r}-1\right) / 2} \mathbf{K}_{l q}^{p \sigma}(t, \tau)\right| \leq \mathrm{const}(t-\tau)^{(1+\alpha) / 2-1}, \quad 0 \leq \tau<t \leq T, \\
\left|\Delta_{t} D_{t}^{\left(d_{r}-1\right) / 2} \mathbf{K}_{l q}^{p \sigma}(t, \tau)\right| \leq \mathrm{const}(\Delta t)^{(1+\alpha) / 2}(t-\tau)^{\mu-1},
\end{gathered}
$$

$0 \leq \tau<t \leq t+\Delta t \leq T, \mu=\min \{\alpha / 2,1-\alpha / 2\}$.

From (22) and (23) it follows that the functions $D_{t}^{\left(d_{r}-1\right) / 2} \mathbf{K}_{l q}^{p \sigma}$ satisfy the assumptions of Lemma 6 of [12], and therefore

$$
\begin{gathered}
\left|\mathcal{R}_{1 / 2}\left[D_{t}^{\left(d_{r}-1\right) / 2} \mathbf{K}_{l q}^{p \sigma}(t, \tau)\right]\right| \leq \mathrm{const}(t-\tau)^{\alpha / 2-1}, \quad 0 \leq \tau<t \leq T, \\
\left|\Delta_{t} \mathcal{R}_{1 / 2}\left[D_{t}^{\left(d_{r}-1\right) / 2} \mathbf{K}_{l q}^{p \sigma}(t, \tau)\right]\right| \leq \mathrm{const}(\Delta t)^{\beta / 2}(t-\tau)^{\mu-1},
\end{gathered}
$$

$0 \leq \tau<t \leq t+\Delta t \leq T, 0<\beta \leq \alpha \leq 1, \mu=\min \{\alpha / 2,1-\alpha / 2\}$.

Combining (20), (21), (24) and (25), we arrive at

$$
\left|\overline{\mathbf{K}}_{l q}^{p \sigma}(t, \tau)\right| \leq \operatorname{const}(t-\tau)^{\alpha / 2-1}, \quad 0 \leq \tau<t \leq T,
$$




$$
\left|\Delta_{t} \overline{\mathbf{K}}_{l q}^{p \sigma}(t, \tau)\right| \leq \mathrm{const}(\Delta t)^{\beta / 2}(t-\tau)^{\mu-1}, \quad 0 \leq \tau<t \leq t+\Delta t \leq T,
$$

$p, \sigma=1,2, l, q=1, \ldots, l_{0}, 0<\beta \leq \alpha \leq 1, \mu=\min \{\alpha / 2,1-\alpha / 2\}$.

Now, we examine the function $\overline{\mathbf{g}}_{l}^{p}$ given by (19). If $d_{r}$ is even, by (A.5) the function $\overline{\mathbf{g}}_{l}^{p}$ satisfies the assumptions of Lemma 5 of [12] with $N=d_{r} / 2$, and so

$$
\overline{\mathbf{g}}_{l}^{p}(t)=D_{t}^{d_{r} / 2} \mathbf{g}_{l}^{p}(t), \quad 0 \leq \tau<t \leq T .
$$

If $d_{r}$ is odd, by (A.5), $\overline{\mathbf{g}}_{l}^{p}$ satisfies the assumptions of that lemma with $N=\left(d_{r}-1\right) / 2$, and thus

$$
\overline{\mathbf{g}}_{l}^{p}(t)=\mathbf{R}_{1 / 2}\left[D_{t}^{\left(d_{r}-1\right) / 2} \mathbf{g}_{l}^{p}(t)\right], \quad 0 \leq \tau<t \leq T .
$$

Hence

$$
\overline{\mathbf{g}}_{l}^{p}(t)= \begin{cases}D_{t}^{d_{r} / 2} \mathbf{g}_{l}^{p}(t) & \text { if } d_{r} \text { is even, } \\ \mathbf{R}_{1 / 2}\left[D_{t}^{\left(d_{r}-1\right) / 2} \mathbf{g}_{l}^{p}(t)\right] & \text { if } d_{r} \text { is odd, }\end{cases}
$$

$\left(d_{r}=n-r_{l}^{p}+2 m+1, p=1,2, l=1, \ldots, l_{0}, 0<t \leq T\right)$.

From (28) and (A.5) in case (i) we obtain

$$
\left|\Delta_{t} \overline{\mathbf{g}}_{l}^{p}(t)\right| \leq \operatorname{const}(\Delta t)^{\alpha / 2}, \quad 0 \leq t<t+\Delta t \leq T, \quad \overline{\mathbf{g}}_{l}^{p}(0)=0 .
$$

In case (ii) we have

$$
\begin{aligned}
\left|\Delta_{t} D^{\left(d_{r}-1\right) / 2} \mathbf{g}_{l}^{p}(t)\right| & \leq \operatorname{const}(\Delta t)^{(1+\alpha) / 2}, \quad 0 \leq t<t+\Delta t \leq T, \\
D_{t}^{\left(d_{r}-1\right) / 2} \mathbf{g}_{l}^{p}(0) & =0,
\end{aligned}
$$

hence, by Lemma 2 of [16], we also get (29).

It remains to investigate the function $\overline{\boldsymbol{z}}_{l}^{p}$ given by (18). Using (5) and Lemma 5 of [12], we obtain

$$
\overline{\boldsymbol{z}}_{l}^{p}(t)= \begin{cases}D_{t}^{d_{r} / 2} \boldsymbol{z}_{l}^{p}(t) & \text { if } d_{r} \text { is even, } \\ \mathbf{R}_{1 / 2}\left[D_{t}^{\left(d_{r}-1\right) / 2} \boldsymbol{z}_{l}^{p}(t)\right] & \text { if } d_{r} \text { is odd },\end{cases}
$$

$\left(d_{r}=n-r_{l}^{p}+2 m+1, p=1,2, l=1, \ldots, l_{0}, 0<t \leq T\right)$; hence, by Lemma 8 of [12], we find

$$
\left|\Delta_{t} \overline{\boldsymbol{z}}_{l}^{p}(t)\right| \leq \mathrm{const}(\Delta t)^{\alpha / 2}, \quad 0 \leq t<t+\Delta t \leq T, \quad \overline{\boldsymbol{z}}_{l}^{p}(0)=0 .
$$

Now, we return to system (16). Multiplying both sides by $[\mathbf{a}(t)]^{-\left(n-r_{l}^{p}\right) / 2}$ we obtain

$$
\sum_{q=1}^{l_{0}} c_{l q}^{p} \varphi_{q}^{p}(t)+\sum_{\sigma=1}^{2} \sum_{q=1}^{l_{0}} \int_{0}^{t} \overline{\mathbf{K}}_{l q}^{p \sigma}(t, \tau) \varphi_{q}^{\sigma}(\tau) d \tau+\overline{\overline{\mathbf{z}}}_{l}^{p}(t)=\overline{\overline{\mathbf{g}}}_{l}^{p}(t)
$$

$\left(p=1,2, l=1, \ldots, l_{0}, 0<t \leq T\right)$, where

$$
\begin{aligned}
\overline{\mathbf{\mathbf { K }}}_{l q}^{p \sigma}(t, \tau) & =[\mathbf{a}(t)]^{-\left(n-r_{l}^{p}\right) / 2} \overline{\mathbf{K}}_{l q}^{p \sigma}(t, \tau), & \overline{\overline{\mathbf{z}}}_{l}^{p}(t) & =[\mathbf{a}(t)]^{-\left(n-r_{l}^{p}\right) / 2} \overline{\boldsymbol{z}}_{l}^{p}(t), \\
\overline{\overline{\mathbf{g}}}_{l}^{p}(t) & =[\mathbf{a}(t)]^{-\left(n-r_{l}^{p}\right) / 2} \overline{\mathbf{g}}_{l}^{p}(t), & \mathbf{a}(t) & =a_{n+2, m}\left(\chi_{p}(t), t\right) .
\end{aligned}
$$


Using assumptions (A.1), (A.2) it can be proved that the functions $\overline{\mathbf{K}}_{l q}^{p \sigma}$, $\overline{\overline{\mathbf{z}}}_{l}^{p}$ and $\overline{\overline{\mathbf{g}}}_{l}^{p}$ satisfy the estimates (26), (27), (29) and (30) respectively.

Now, we treat system (31) as an algebraic system with respect to the functions $\varphi_{q}^{p}, p=1,2, q=1, \ldots, l_{0}$. Its determinant is of the form

Hence, in view of (9), we have

$$
\mathbf{W}=\left|\begin{array}{ccccc}
c_{11}^{p} & 0 & 0 & \ldots & 0 \\
c_{21}^{p} & c_{22}^{p} & 0 & \ldots & 0 \\
c_{31}^{p} & c_{32}^{p} & c_{33}^{p} & \ldots & 0 \\
\vdots & \vdots & \vdots & \ddots & \vdots \\
c_{l_{0}, 1}^{p} & c_{l_{0}, 2}^{p} & c_{l_{0}, 3}^{p} & \ldots & c_{l_{0}, l_{0}}^{p}
\end{array}\right| .
$$

$$
\mathbf{W}=c_{11}^{p} c_{22}^{p} \ldots c_{l_{0}, l_{0}}^{p}=(-1)^{n l_{0}-\left(r_{1}^{p}+r_{2}^{p}+\ldots+r_{l_{0}}^{p}\right)}(\sqrt{\pi})^{l_{0}} \neq 0
$$

on one of the curves $\chi_{p}$ (see $\S 1$ ) and

$$
\mathbf{W}=c_{11}^{p} c_{22}^{p} \ldots c_{l_{*}-1, l_{*}-1}^{p} c_{l_{*}+1, l_{*}+1}^{p} \ldots c_{l_{0}, l_{0}}^{p} \neq 0
$$

on the other. Cramer's formulae yield

$$
\varphi_{q}^{p}(t)+\sum_{\sigma=1}^{2} \sum_{q=1}^{l_{0}} \int_{0}^{t} \widetilde{\mathbf{K}}_{l q}^{p \sigma}(t, \tau) \varphi_{q}^{\sigma}(\tau) d \tau+\widetilde{\boldsymbol{z}}_{l}^{p}(t)=\widetilde{\mathbf{g}}_{l}^{p}(t),
$$

where

$$
\begin{aligned}
\widetilde{\mathbf{K}}_{l q}^{p \sigma}(t, \tau) & =\sum_{v=1}^{l_{0}} A_{l v}^{p} \overline{\overline{\mathbf{K}}}_{v q}^{p \sigma}(t, \tau), & \widetilde{\boldsymbol{z}}_{l}^{p}(t) & =\sum_{v=1}^{l_{0}} A_{l v}^{p} \overline{\overline{\mathbf{z}}}_{v}^{p}(t), \\
\widetilde{\mathbf{g}}_{l}^{p}(t) & =\sum_{v=1}^{l_{0}} A_{l v}^{p} \overline{\overline{\mathbf{g}}}_{v}^{p}(t), & A_{l v}^{p} & =C_{l v}^{p} / \mathbf{W},
\end{aligned}
$$

$p=1,2, l=1, \ldots, l_{0}, 0<t \leq T\left(C_{l v}^{p}\right.$ denotes the algebraic complement of $c_{l v}^{p}$ in $\left.\mathbf{W}\right)$.

It is easy to see that $\widetilde{\mathbf{K}}_{l q}^{p \sigma}, \widetilde{\boldsymbol{z}}_{l}^{p}$ and $\widetilde{\mathbf{g}}_{l}^{p}$ satisfy the same estimates as $\overline{\mathbf{\mathbf { K }}}_{l q}^{p \sigma}, \overline{\overline{\boldsymbol{z}}}_{l}^{p}$ and $\overline{\mathbf{g}}_{l}^{p}$ respectively. Thus, (32) is a system of second-type Volterra integral equations with weak singularities and hence it has a solution of the form

$$
\varphi_{l}^{p}(t)=\widetilde{\mathbf{g}}_{l}^{p}(t)-\widetilde{\boldsymbol{z}}_{l}^{p}(t)+\sum_{\sigma=1}^{2} \sum_{q=1}^{l_{0}} \int_{0}^{t} \boldsymbol{\mathcal { K }}_{l q}^{p \sigma}(t, \tau)\left[\widetilde{\mathbf{g}}_{q}^{\sigma}(\tau)-\widetilde{\boldsymbol{z}}_{q}^{\sigma}(\tau)\right] d \tau,
$$

where $\mathcal{K}_{l q}^{p \sigma}$ denote the resolvent kernels of the $\widetilde{\mathbf{K}}_{l q}^{p \sigma}, p, \sigma=1,2, l, q=$ $1, \ldots, l_{0}$. Moreover, the estimates (26), (27), (29) and (30) imply

$$
\left|\Delta_{t} \varphi_{l}^{p}(t)\right| \leq \operatorname{const}(\Delta t)^{\beta / 2}, \quad \varphi_{l}^{p}(0)=0
$$

$\left(p=1,2, l=1, \ldots, l_{0}, 0 \leq t<t+\Delta t \leq T, 0<\beta \leq \alpha \leq 1\right)$. 
3.2. Case 3). Without losing generality we may assume that on both the curves $\chi_{p}, l_{0}-1$ conditions are posed given by the operators $\mathbf{B}_{l}^{p}, p=1,2$, $l=1, \ldots, l_{0}-1$, with $0 \leq r_{1}^{p}<r_{2}^{p}<\ldots<r_{l_{0}-1}^{p}<n+1$, and moreover, one more condition given by $\mathbf{B}_{l_{0}}^{p}$ with $r_{l_{0}}^{1}=n+1$ is posed on $\chi_{1}$.

Now, we rewrite formula (4) in a form more suitable for further considerations:

$$
\begin{aligned}
u(x, t)= & \int_{0}^{t} \Lambda_{n+1}\left(x, t ; \chi_{1}(\tau), \tau\right) \varphi_{l_{0}}^{1}(\tau) d \tau \\
& +\sum_{\sigma=1}^{2} \sum_{q=1}^{l_{0}-1} \int_{0}^{t} \Lambda_{r_{q}^{\sigma}}\left(x, t ; \chi_{\sigma}(\tau), \tau\right) \varphi_{q}^{\sigma} d \tau+\mathbf{Z}_{\mathbf{S}_{T}}(x, t),
\end{aligned}
$$

where the functions $\Lambda_{r_{q}^{\sigma}}$ for $\sigma=1,2, q=1, \ldots, l_{0}-1$ are defined by formula (7) of [12] and

$$
\Lambda_{n+1}(x, t ; y, \tau)=\Lambda_{r_{*}^{1}}(x, t ; y, \tau)
$$

$\left((x, t),(y, \tau) \in \overline{\mathbf{S}}_{T}\right)$, where $r_{*}^{1}$ is a positive integer with $0 \leq r_{*}^{1} \leq n, r_{*}^{1} \neq r_{l}^{1}$ for $l=0,1, \ldots, l_{0}-1$.

Applying to both sides of (35) the operator $\mathbf{B}_{l_{0}}^{1}$ given by (3), we get

$$
\begin{aligned}
\mathbf{B}_{l_{0}}^{1} u(x, t)= & \int_{0}^{t} \mathbf{B}_{l_{0}}^{1} \Lambda_{r_{*}^{1}}\left(x, t ; \chi_{1}(\tau), \tau\right) \varphi_{l_{0}}^{1}(\tau) d \tau \\
& +\sum_{\sigma=1}^{2} \sum_{q=1}^{l_{0}-1} \int_{0}^{t} \mathbf{B}_{l_{0}}^{1} \Lambda_{r_{q}^{\sigma}}\left(x, t ; \chi_{1}(\tau), \tau\right) \varphi_{q}^{\sigma}(\tau) d \tau+\mathbf{B}_{l_{0}}^{1} \mathbf{Z}_{\mathbf{S}_{T}}(x, t) .
\end{aligned}
$$

By (5) and Lemma 2 of [12] we can write

$\mathbf{B}_{l_{0}}^{1} \Lambda_{r_{*}^{1}}\left(x, t ; \chi_{1}(\tau), \tau\right)=\mathbf{P}_{m}\left[D_{x} \omega^{\chi_{1}(\tau), \tau}\left(x, t ; \chi_{1}(\tau), \tau\right)\right]+\mathbf{B}_{l_{0}}^{1} \bar{w}_{r_{*}^{1}}\left(x, t ; \chi_{1}(\tau), \tau\right)$ $\left((x, t) \in \overline{\mathbf{S}}_{T}\right)$. Consider the integral

$$
\mathbf{J}_{m}(x, t)=\int_{0}^{t} \mathbf{P}_{m}\left[D_{x} \omega^{\chi_{1}(\tau), \tau}\left(x, t ; \chi_{1}(\tau), \tau\right)\right] \varphi_{l_{0}}^{1}(\tau) d \tau \quad\left(m \in \mathbb{N}_{0}\right) .
$$

We investigate its behaviour as $x \rightarrow \chi_{1}(t),(x, t) \in \mathbf{S}_{T}$. For $m=0$ we have

$$
\mathbf{J}_{0}(x, t)=\int_{0}^{t} D_{x} \omega^{\chi_{1}(\tau), \tau}\left(x, t ; \chi_{1}(\tau), \tau\right) \varphi_{l_{0}}^{1}(\tau) d \tau .
$$

This is a heat potential of second kind which has the following property ([7], p. 1085):

$$
\lim _{x \rightarrow \chi_{1}(t)} \mathbf{J}_{0}(x, t)=-\sqrt{\frac{\pi}{\mathbf{a}(t)}} \varphi_{l_{0}}^{1}(t)+\mathbf{J}_{0}\left(\chi_{1}(t), t\right), \quad(x, t) \in \mathbf{S}_{T},
$$

where $\mathbf{a}(t)=a_{n+2,0}\left(\chi_{1}(t), t\right)$. 
For $m>0$ the integral $\mathbf{J}_{m}$ can be written in the form

$$
\mathbf{J}_{m}(x, t)=\int_{0}^{t}\left[\int_{\tau}^{t} \frac{\left(t-\zeta_{m}\right)^{m-1}}{(m-1) !} D_{x} \omega^{\chi_{1}(\tau), \tau}\left(x, \zeta_{m} ; \chi_{1}(\tau), \tau\right) d \zeta_{m}\right] \varphi_{l_{0}}^{1}(\tau) d \tau .
$$

It follows that

$$
\mathbf{J}_{m}(x, t)=\int_{0}^{t} \frac{\left(t-\zeta_{m}\right)^{m-1}}{(m-1) !} \mathbf{J}_{0}\left(x, \zeta_{m}\right) d \zeta_{m},
$$

and hence, by (38), we obtain

(39) $\lim _{x \rightarrow \chi_{1}(t)} \mathbf{J}_{m}(x, t)=-\int_{0}^{t} \frac{\left(t-\zeta_{m}\right)^{m-1}}{(m-1) !} \sqrt{\frac{\pi}{\mathbf{a}(t)}} \varphi_{l_{0}}^{1}\left(\zeta_{m}\right) d \zeta_{m}+\mathbf{J}_{m}\left(\chi_{1}(t), t\right)$ $\left((x, t) \in \mathbf{S}_{T}, m \in \mathbb{N}\right)$.

Making use of the definition of the operator $\mathbf{I}_{\kappa}$ (see (25) in [12]), formulae (38) and (39) can be written in the form

$$
\lim _{x \rightarrow \chi_{1}(t)} \mathbf{J}_{m}(x, t)=-\mathbf{I}_{m}\left[\sqrt{\frac{\pi}{\mathbf{a}(t)}} \varphi_{l_{0}}^{1}(t)\right]+\mathbf{J}_{m}\left(\chi_{1}(t), t\right)
$$

$\left((x, t) \in \mathbf{S}_{T}, m \in \mathbb{N}_{0}\right)$, where $\mathbf{a}(t)=a_{n+2, m}\left(\chi_{1}(t), t\right)$.

Passing to the limit $x \rightarrow \chi_{1}(t)$ in (37), we have

$$
\begin{aligned}
\mathbf{g}_{l_{0}}^{1}(t)= & -\mathbf{I}_{m}\left[\sqrt{\frac{\pi}{\mathbf{a}(t)}} \varphi_{l_{0}}^{1}(t)\right]+\int_{0}^{t} \mathbf{K}_{l_{0} l_{0}}^{11}(t, \tau) \varphi_{l_{0}}^{1}(\tau) d \tau \\
& +\sum_{\sigma=1}^{2} \sum_{q=1}^{l_{0}-1} \int_{0}^{t} \mathbf{K}_{l_{0} q}^{1 \sigma}(t, \tau) \varphi_{q}^{\sigma}(\tau) d \tau+\boldsymbol{z}_{l_{0}}^{1}(t),
\end{aligned}
$$

where $\mathbf{K}_{l_{0} l_{0}}^{11}(t, \tau)=\mathbf{B}_{l_{0}}^{1} \Lambda_{r_{*}^{1}}\left(\chi_{1}(t), t ; \chi_{1}(\tau), \tau\right), \mathbf{K}_{l_{0} q}^{1 \sigma}(t, \tau)=\mathbf{B}_{l_{0}}^{1} \Lambda_{r_{q}^{\sigma}}\left(\chi_{1}(t), t\right.$; $\left.\chi_{\sigma}(\tau), \tau\right), \sigma=1,2, q=1, \ldots, l_{0}-1,0<t \leq T$, the operators $\mathbf{B}_{l_{0}}^{1}$ are defined by formula (34) of [12] and the functions $\boldsymbol{z}_{l_{0}}^{1}$ are given by relation (42) of [12].

Applying $\mathbf{R}_{1 / 2}^{2 m}$ to both sides of (41), by Lemmas 4 and 5 of [12], we obtain

$$
\begin{aligned}
& -\sqrt{\frac{\pi}{\mathbf{a}(t)}} \varphi_{l_{0}}^{1}(t)+\int_{0}^{t} \overline{\mathbf{K}}_{l_{0} l_{0}}^{11}(t, \tau) \varphi_{l_{0}}^{1}(\tau) d \tau \\
& +\sum_{\sigma=1}^{2} \sum_{q=1}^{l_{0}-1} \int_{0}^{t} \overline{\mathbf{K}}_{l_{0} q}^{1 \sigma}(t, \tau) \varphi_{q}^{\sigma}(\tau) d \tau+\overline{\mathbf{z}}_{l_{0}}^{1}(t)=\overline{\mathbf{g}}_{l_{0}}^{1}(t), \quad 0<t \leq T,
\end{aligned}
$$

where $\overline{\mathbf{K}}_{l_{0} l_{0}}^{11}(t, \tau)=D_{t}^{m} \mathbf{K}_{l_{0} l_{0}}^{11}(t, \tau), \overline{\mathbf{K}}_{l_{0} q}^{1 \sigma}(t, \tau)=D_{t}^{m} \mathbf{K}_{l_{0} q}^{1 \sigma}(t, \tau), \overline{\boldsymbol{z}}_{l_{0}}^{1}(t)=$ $D_{t}^{m} \boldsymbol{z}_{l_{0}}^{1}(t), \overline{\mathbf{g}}_{l_{0}}^{1}(t)=D_{t}^{m} \mathbf{g}_{l_{0}}^{1}(t), \sigma=1,2, q=1, \ldots, l_{0}-1$. 
Using Theorem 2 of [12] we find the estimates

$$
\begin{array}{rll}
\text { (43) } & \left|\overline{\mathbf{K}}_{l_{0} l_{0}}^{11}(t, \tau)\right| \leq \mathrm{const}(t-\tau)^{\alpha / 2-1}, & 0 \leq \tau<t \leq T, \\
\text { (44) } & \left|\overline{\mathbf{K}}_{l_{0} q}^{1 \sigma}(t, \tau)\right| \leq \mathrm{const}(t-\tau)^{\alpha / 2-1}, & 0 \leq \tau<t \leq T, \\
\text { (45) } & \left|\Delta_{t} \overline{\mathbf{K}}_{l_{0} l_{0}}^{11}(t, \tau)\right| \leq \mathrm{const}(\Delta t)^{\beta / 2}(t-\tau)^{\mu-1}, & 0 \leq \tau<t \leq t+\Delta t \leq T, \\
\text { (46) } & \left|\Delta_{t} \overline{\mathbf{K}}_{l_{0} q}^{1 \sigma}(t, \tau)\right| \leq \mathrm{const}(\Delta t)^{\beta / 2}(t-\tau)^{\mu-1}, & 0 \leq \tau<t \leq t+\Delta t \leq T,
\end{array}
$$

where $\sigma=1,2, q=1, \ldots, l_{0}-1,0<\beta \leq \alpha \leq 1, \mu=\min \{\alpha / 2,1-\alpha / 2\}$.

Similarly, using Lemma 9 of [12], we have

$$
\left|\Delta_{t} \bar{z}_{l_{0}}^{1}(t)\right| \leq \operatorname{const}(\Delta t)^{\alpha / 2}, \quad 0 \leq t<t+\Delta t \leq T, \quad \bar{z}_{l_{0}}^{1}(0)=0,
$$

moreover, in view of assumption (A.5), we get

$$
\left|\Delta_{t} \overline{\mathbf{g}}_{l_{0}}^{1}(t)\right| \leq \operatorname{const}(\Delta t)^{\alpha / 2}, \quad 0 \leq t<t+\Delta t \leq T, \quad \overline{\mathbf{g}}_{l_{0}}^{1}(0)=0 .
$$

Observe that equation (42) can be written in the form

$$
\begin{aligned}
\varphi_{l_{0}}^{1}(t)+\int_{0}^{t} \widetilde{\mathbf{K}}_{l_{0} l_{0}}^{11}(t, \tau) \varphi_{l_{0}}^{1}(\tau) d \tau \\
\quad+\sum_{\sigma=1}^{2} \sum_{q=1}^{l_{0}-1} \int_{0}^{t} \widetilde{\mathbf{K}}_{l_{0} q}^{1 \sigma}(t, \tau) \varphi_{q}^{\sigma}(\tau) d \tau+\widetilde{\boldsymbol{z}}_{l_{0}}^{1}(t)=\widetilde{\mathbf{g}}_{l_{0}}^{1}(t),
\end{aligned}
$$

where $\widetilde{\mathbf{K}}_{l_{0} l_{0}}^{11}(t, \tau)=-\sqrt{\mathbf{a}(t) / \pi} \cdot \overline{\mathbf{K}}_{l_{0} l_{0}}^{11}(t, \tau), \widetilde{\mathbf{K}}_{l_{0} q}^{1 \sigma}(t, \tau)=-\sqrt{\mathbf{a}(t) / \pi} \cdot \overline{\mathbf{K}}_{l_{0} q}^{1 \sigma}(t, \tau)$, $\widetilde{\boldsymbol{z}}_{l_{0}}^{1}(t)=-\sqrt{\mathbf{a}(t) / \pi} \cdot \overline{\boldsymbol{z}}_{l_{0}}^{1}(t), \widetilde{\mathbf{g}}_{l_{0}}^{1}(t)=-\sqrt{\mathbf{a}(t) / \pi} \cdot \overline{\mathbf{g}}_{l_{0}}^{1}(t), \sigma=1,2, q=$ $1, \ldots, l_{0}-1,0<t \leq T$.

From assumptions (A.1) and (A.2) it follows that $\overline{\mathbf{K}}_{l_{0} l_{0}}^{11}, \overline{\overline{\mathbf{K}}}_{l_{0} q}^{1 \sigma}, \overline{\overline{\mathbf{z}}}_{l_{0}}^{1}$ and $\overline{\mathbf{g}}_{l_{0}}$ satisfy inequalities (43)-(48) respectively. This means that if we treat the functions $\varphi_{q}^{\sigma}, \sigma=1,2, q=1, \ldots, l_{0}-1$, as parameters, then (49) is a second-kind Volterra equation with respect to $\varphi_{l_{0}}^{1}$. Because the singularity of the kernel of this equation is weak one can solve it.

Imposing on the function $u$, given by formula (35), the remaining boundary conditions (3) given by the operators $\mathbf{B}_{1}^{p}, \mathbf{B}_{2}^{p}, \ldots, \mathbf{B}_{l_{0}-1}^{p}$ with $0 \leq r_{1}^{p}<$ $r_{2}^{p}<\ldots<r_{l_{0}-1}^{p}<n+1\left(p=1,2, l_{0}=[(n+3) / 2]\right)$, we obtain the following system of integral equations:

$$
\begin{aligned}
\sum_{\sigma=1}^{2} \sum_{q=1}^{l_{0}-1} \int_{0}^{t} \mathbf{B}_{l}^{p} & \Lambda_{r_{q}^{\sigma}}\left(\chi_{p}(t), t ; \chi_{p}(\tau), \tau\right) \varphi_{q}^{\sigma}(\tau) d \tau \\
& +\int_{0}^{t} \mathbf{B}_{l}^{p} \Lambda_{r_{*}^{1}}\left(\chi_{p}(t), t ; \chi_{1}(\tau), \tau\right) \varphi_{l_{0}}^{1}(\tau) d \tau+\boldsymbol{z}_{l}^{p}(t)=\mathbf{g}_{l}^{p}(t),
\end{aligned}
$$

$p=1,2, l=1, \ldots, l_{0}-1,0<t \leq T$. 
System (50) is a system of first-kind Volterra integral equations with $2\left(l_{0}-1\right)$ equations and $2\left(l_{0}-1\right)$ unknown functions $\varphi_{q}^{\sigma}, \sigma=1,2, q=$ $1, \ldots, l_{0}-1$. Now, we apply to system (50) the method presented in subsection 3.1 to obtain

$$
\begin{aligned}
\varphi_{l}^{p}(t)+\sum_{\sigma=1}^{2} \sum_{q=1}^{l_{0}-1} \int_{0}^{t} \widetilde{\mathbf{K}}_{l q}^{p \sigma}(t, \tau) \varphi_{q}^{\sigma}(\tau) d \tau \\
=\int_{0}^{t} \widetilde{\mathbf{K}}_{l_{0} l_{0}}^{11}(t, \tau) \varphi_{l_{0}}^{1}(\tau) d \tau-\widetilde{\mathbf{g}}_{l}^{p}(t)-\widetilde{\boldsymbol{z}}_{l}^{p}(t),
\end{aligned}
$$

$p=1,2, l=1, \ldots, l_{0}-1,0<t \leq T$.

The functions $\widetilde{\mathbf{K}}_{l q}^{p \sigma}, \widetilde{\mathbf{g}}_{l}^{p}$ and $\widetilde{\boldsymbol{z}}_{l}^{p}$ satisfy inequalities (26), (27), (29) and (30), respectively, thus (51) is a system of second-kind Volterra integral equations with weak singularities.

Finally, we are able to find a solution of system (49), (51) in the form

$$
\begin{aligned}
\varphi_{l}^{p}(t)= & \overline{\mathbf{g}}_{l}^{p}(t)-\overline{\mathbf{z}}_{l}^{p}(t) \\
& +\sum_{\sigma=1}^{2} \sum_{q=1}^{l_{0}-1} \int_{0}^{t}\left[\mathcal{K}_{l q}^{p \sigma}(t, \tau)-\mathcal{K}_{l_{0} l_{0}}^{11}(t, \tau)\right]\left[\overline{\mathbf{g}}_{q}^{\sigma}(\tau)-\overline{\boldsymbol{z}}_{q}^{\sigma}(\tau)\right] d \tau
\end{aligned}
$$

$\left(l=1, \ldots, l_{0}\right.$ for $p=1, l=1, \ldots, l_{0}-1$, for $\left.p=2\right)$, where $\mathcal{K}_{l q}^{p \sigma}$ and $\mathcal{K}_{l_{0} l_{0}}^{11}$ are the resolvent kernels of $\widetilde{\mathbf{K}}_{l q}^{p \sigma}$ and $\widetilde{\mathbf{K}}_{l_{0} l_{0}}^{11}$, respectively. Furthermore, by (26) $-(27),(29)-(30)$ and $(43)-(48)$ we obtain

$$
\left|\Delta_{t} \varphi_{l}^{p}(t)\right| \leq \operatorname{const}(\Delta t)^{\beta / 2}, \quad 0 \leq t<t+\Delta t \leq T, \quad \varphi_{l}^{p}(0)=0
$$

$\left(p=1,2, l=1, \ldots, l_{0}-1\right)$, where $0<\beta \leq \alpha \leq 1$.

As a result of the foregoing considerations we can formulate the following theorem:

THEOREM 1. If assumptions (A.1)-(A.6) are satisfied then there exists a solution $u$ of the problem (1)-(3). It is given by relation (4), where the functions $\varphi_{q}^{\sigma}$ are defined by formula (33) in case 1); by a formula similar to (33) in case 2) and then they satisfy inequality (34); by formula (52) in case 3 ); and by a formula similar to (52) in case 4) and then they satisfy inequality (53).

\section{References}

[1] E. A. Baderko, On solvability of boundary-value problems for parabolic equations of higher order in curvilinear domains, Differentsial'nye Uravneniya 12 (1976), 17821792 (in Russian).

[2] Z. D. Dubl'a, Boundary-value problems for differential equations in unbounded domains, ibid. 10 (1974), 159-161 (in Russian). 
[3] Z. D. Dubl'a, On the Dirichlet problem for a class of equations of third order, ibid. 13 (1977), 50-55 (in Russian).

[4] T. D. Dzhuraev, Boundary-Value Problems for Equations of Mixed and MixedComposite Types, FAN, Tashkent, 1979 (in Russian).

[5] T. D. Dzhuraev and M. Mamazhanov, On a class of boundary-value problems for equations of third order containing the operator of heat conduction, Izv. Akad. Nauk UzSSR 1985 (2), 22-26 (in Russian).

[6] M. Hanin, Propagation of an aperiodic wave in a compressible viscous medium, J. Math. Phys. 36 (1957), 133-150.

[7] L. I. Kamynin, The method of heat potentials for parabolic equations with discontinuous coefficients, Sibirsk. Mat. Zh. 4 (1963), 1071-1105 (in Russian).

[8] R. Nardini, Soluzione di un problema al contorno della magneto-idrodinamica, Ann. Mat. Pura Appl. 35 (1953), 269-290.

[9] - Sul comportamento asintotico della soluzione di un problema al contorno della magneto-idrodinamica, Rend. Accad. Naz. Lincei 16 (1954), 225-231, 341-348, 365-366.

[10] B. Pini, Un problema di valori al contorno per un'equazione a derivate parziali del terzo ordine con parte principale di tipo composito, Rend. Sem. Fac. Sci. Univ. Cagliari 27 (1957), 114-135.

[11] J. Popiołek, The Cauchy problem for a higher-order partial differential equation, Izv. Akad. Nauk UzSSR 1 (1989), 25-30 (in Russian).

[12] - , Properties of some integrals related to partial differential equations of order higher than two, this issue, 129-138.

[13] A. S. Rustamov, A mixed problem for the equation of composite type with variable coefficients, Differentsial'nye Uravneniya 18 (1982), 1794-1804 (in Russian).

[14] S. N. Salikhov, On a boundary-value problem for a partial differential equation with multiple characteristics, Izv. Akad. Nauk UzSSR 1983 (5), 29-33 (in Russian).

[15] Ya. S. Sharifbaev, On some boundary-value problems for equations of third order with the heat conduction operator in the principal part, ibid. 1975 (1), 45-48 (in Russian).

[16] J. Urbanowicz, On a certain non-linear contact problem for a one-dimensional parabolic equation of second order, Demonstratio Math. 16 (1983), 61-83.

[17] S. S. Vojt, Propagation of initial waves in a viscous gas, Uchen. Zap. MTU 172 (1954), 125-142 (in Russian).

Institute of Mathematics

Warsaw University, Białystok Branch

Akademicka 2

15-267 Białystok, Poland

E-mail: popiolek@math.uw.bialystok.pl 